\title{
Temperature Measurement Method Based on Riesz Transform Method
}

\author{
Sara Zada, Salah Darfi, Vamara Dembele, Said Rachafi, and Abdelkarim Nassim \\ Laboratoire Instrumentation de Mesure et Contrôle (IMC), Université Chouaib Doukkali (UCD), Faculté des Sciences El Jadida (FSJ), \\ BP 20, 24000 El Jadida, Morocco
}

Correspondence should be addressed to Abdelkarim Nassim; nassim.a@ucd.ac.ma

Received 1 October 2013; Accepted 7 November 2013

Academic Editors: L. Carretero and S. Gallego

Copyright (c) 2013 Sara Zada et al. This is an open access article distributed under the Creative Commons Attribution License, which permits unrestricted use, distribution, and reproduction in any medium, provided the original work is properly cited.

\begin{abstract}
A method to measure the temperature of a metal plate is presented using a Riesz transform method and the monogenic signal to extract the optical phase distribution from a fringe pattern from which one can get the unknown temperature. The performance of this method is evaluated by the RFSIM metric obtained from the 2nd-order Riesz transform. A phase distribution with a good accuracy is provided.
\end{abstract}

\section{Introduction}

The measurement of temperature field is of great significance to scientific research and national economic development. This is because the measurement of thermal physical quantities is widely used in the implementation and control of industrial processes such as power, aerospace, chemical industry, and oil refining, among others [1]. Temperatures are usually measured by inserting thermocouples into tested fields and entire temperature fields are reconstructed using readings from the thermocouples. However, this method has drawbacks. Temperatures at different points are not obtained simultaneously and tested fields are disturbed by the thermocouples themselves. In addition, obtained readings need to be compensated against the effect brought about by radiation.

Various optical methods that are full-field, sensitive, and noncontact include holographic interferometry $[1,2]$, speckle shearing interferometry [3], Moiré deflectometry [4], effect mirage [5], and digital speckle pattern interferometry (DSPI) [6], which have been used to measure the temperature.

Digital speckle pattern interferometry is a whole field optical method for noncontact and nondestructive surface analysis. It is now considered as a powerful tool for industrial measurements. It enables full-field measurement of optical phase changes via the acquisition of speckle patterns [7-9].
After acquisition, a simple subtraction is usually performed to obtain a correlation fringe pattern. The greatest challenges in speckle interferometry focus on relating fringe patterns to phase mapping, permitting the direct determination of surface displacement. However, as DSPI fringes are characterized by a strong speckle noise background, a denoising method [10-12] must be used before the phase evaluation. The removal of speckle noise in DSPI is a complex problem which has generated an active area of research.

The aim of this study is the application of the Riesz transform and the monogenic signal to extract the optical phase distribution from a single fringe pattern without the step of speckle denoising from which one can get the unknown temperature. Felsberg and Sommer [13] proposed two-dimensional generalization of the analytic signal called monogenic signal. This representation based on the Riesz transform [14] instead of the Hilbert transform preserves the core properties of the analytic signal that decomposes an image into information about structure. The monogenic signal is characterized by its three independent sizes as local amplitude, local phase, and local orientation. It has proved its usefulness in several applications [15-17].

The paper is organized as follows. Section 2 is devoted to the description of the monogenic phase extraction technique and the RFSIM metrics. In Section 3, a brief description of speckle correlation fringe obtained by digital speckle 


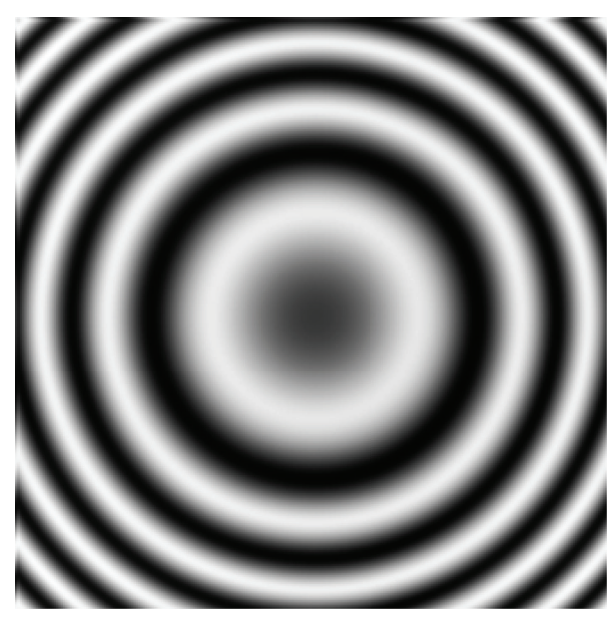

(a)

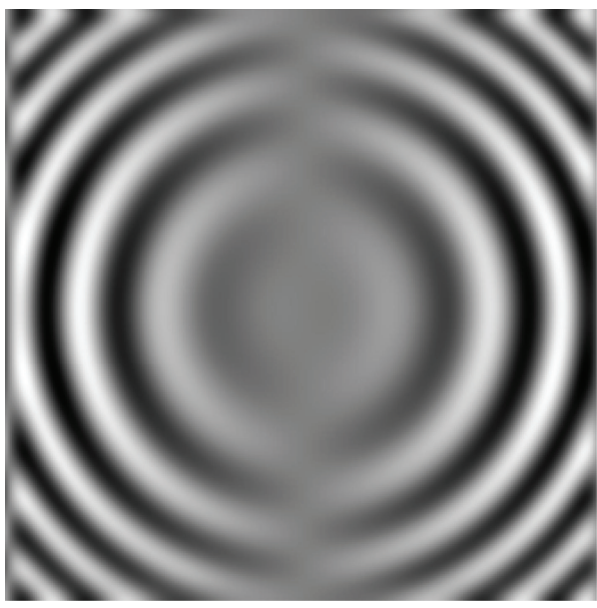

(b)

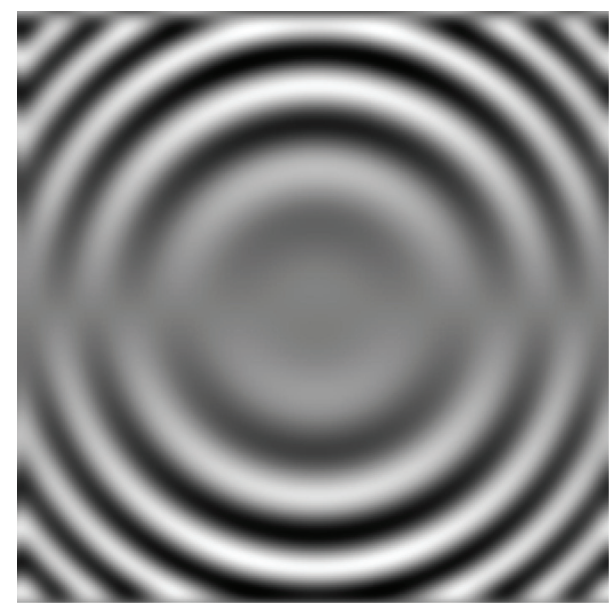

(c)

Figure 1: Simulation results: (a) fringe pattern, (b) Riesz component of the fringe pattern on $x$-axis, and (c) Riesz component of the fringe pattern on $y$-axis.

pattern interferometry (DSPI) technique for temperature measurement is presented. The numerical simulations and results of temperature evaluation are presented in Section 4. Finally a conclusion is given in Section 5 .

\section{The Riesz Transform}

The Riesz transform for an image $p$ is defined by

$$
R(p(x, y))=\left\{\begin{array}{c}
q_{1}(x, y)=R_{x}(x, y) * p(x, y) \\
q_{2}(x, y)=R_{y}(x, y) * p(x, y)
\end{array}\right.
$$

where $*$ stands for the convolution and $p(x, y)$ is the image to be analyzed. $R_{x}$ and $R_{y}$ are the spatial representation of the Riesz kernel, respectively, defined by

$$
\begin{aligned}
& R_{x}(x, y)=\frac{-x}{2 \pi\left(x^{2}+y^{2}\right)^{3 / 2}} \\
& R_{y}(x, y)=\frac{-y}{2 \pi\left(x^{2}+y^{2}\right)^{3 / 2}}
\end{aligned}
$$

The two output images $q_{1}$ and $q_{2}$ are the Riesz transform components on $x$-axis and $y$-axis. The Riesz transform is the natural 2D extension of the Hilbert transform.

2.1. The Monogenic Signal. For an image, the monogenic signal is defined as the combination of the image $p$ and its Riesz transform components:

$$
S_{M}(x, y)=\left(p(x, y), q_{1}(x, y), q_{2}(x, y)\right)^{T} .
$$

From this formulation, the monogenic signal is $3 \mathrm{D}$ vector and we can get the local features as local amplitude, local orientation, local phase, and phase vector.

The local amplitude is defined by

$$
r=\left(p^{2}+q_{1}^{2}+q_{2}^{2}\right)^{1 / 2}
$$

The local orientation with $\theta \in[-\pi / 2, \pi / 2]$ is defined by

$$
\theta=a \tan \left(\frac{q_{2}}{q_{1}}\right)
$$




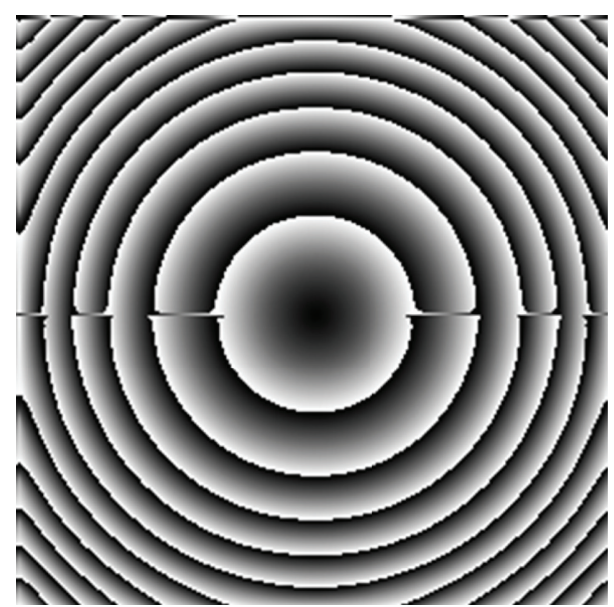

FIGURE 2: The wrapped retrieved phase from Riesz transform method.

The local phase with $\varphi \in[-\pi, \pi]$ is defined by

$$
\varphi=a \tan 2\left(\left(q_{1}^{2}+q_{2}^{2}\right)^{1 / 2}, p\right) .
$$

It is seen that the local orientation, the local phase, and the local amplitude of the image can be conveniently represented via the Riesz transform based on the monogenic signal which decomposes an image into information at each location.

The phase vector $\varphi[15]$ is defined by

$$
\phi=\left(\frac{q}{\left(q_{1}^{2}+q_{2}^{2}\right)^{1 / 2}}\right) \cdot a \tan 2\left(\left(q_{1}^{2}+q_{2}^{2}\right)^{1 / 2}, p\right)
$$

with $q=\left(q_{1}, q_{2}\right)$.

Due to the fact that structural information is related to a given orientation, the phase vector is obtained by the local phase associated with a given local orientation. The phase vector can be interpreted as a rotation vector, in which magnitude corresponds to the phase angle between the image and the monogenic signal.

2.2. RFSIM Metric. The RFSIM metric (Riesz transform based feature similarity) [18] allows judgeing the image quality assessment. This metric is computed by comparing 2nd-order Riesz transform features at key locations between two images (an image with its reference).

The 2nd-order Riesz transform is obtained from the 1storder Riesz transform components $q_{1}$ and $q_{2}$ by

$$
\begin{gathered}
q_{3}=R_{x}\left(q_{1}\right)=R_{x} *\left(R_{x} * p(x, y)\right), \\
q_{4}=R_{x}\left(q_{2}\right)=R_{y}\left(q_{1}\right)=R_{x} *\left(R_{y} * p(x, y)\right), \\
q_{5}=R_{y}\left(q_{2}\right)=R_{y} *\left(R_{y} * p(x, y)\right)
\end{gathered}
$$

with

$$
\begin{aligned}
& q_{1}=R_{x} * p(x, y), \\
& q_{2}=R_{y} * p(x, y) .
\end{aligned}
$$

Let $\left(q_{1}, q_{2}, q_{3}, q_{4}, q_{5}\right)$, the first two are 1st-order Riesz transform coefficients and the last three are 2nd-order coefficients of an image $p$. Similarly, let $\left(q_{1 \text { ref }}, q_{2 \text { ref }}, q_{3 \text { ref }}, q_{4 \text { ref }}, q_{5 \text { ref }}\right)$ : the first two are 1st-order Riesz transform coefficients and the last three are 2 nd-order coefficients of the reference image $p_{\text {ref }}$.

The coefficient RFSIM is defined as

$$
\operatorname{RFSIM}=\prod_{i=1}^{n} D_{i}
$$

where $D_{i}$ is the feature similarity between the two images given by

$$
D_{i}=\frac{\sum \sum d_{i}(x, y) \cdot M(x, y)}{\sum \sum M(x, y)},
$$

where $d_{i}$, the distance of comparison at the corresponding location $(x, y)$ between the two features $q_{i}$ and $q_{\text {iref }}$ ( $i=$ $1, \ldots, 5)$, is formulated by

$$
d_{i}(x, y)=\frac{\left(2 q_{i}(x, y) \cdot q_{\text {iref }}(x, y)\right)}{\left(q_{i}^{2}(x, y)+q_{i \mathrm{ref}}^{2}(x, y)\right)} .
$$

The feature mask $M(x, y)$ is defined as the edge locations of the each image by

$$
M(x, y)=M_{1} \oplus M_{2},
$$

where $\oplus$ is the logical "OR" operation. $M_{1}$ denotes the result of edge detection performed on the image $p$ and $M_{2}$ the result of edge detection on the reference image $p_{\text {ref }}$. The RFSIM metric quantifies how well image details are preserved.

\section{Correlation Fringe Pattern for Temperature Measurement}

3.1. Correlation Fringe Pattern Formation. The basic principle DSPI technique is that the speckle pattern intensity distribution is a function of the relative phases of two interfering plane waves inside each resolution cell of an imaging setup. Displacement of the surface affects the intensity received in each speckle cell on the image. The intensity distribution of a reference speckled image (before displacement) is

$$
I_{1}(x, y)=a(x, y)+b(x, y) \cos \phi_{s}(x, y),
$$

where $a(x, y)$ is the bias intensity, $b(x, y)$ is the visibility, and $\varphi_{s}(x, y)$ is the original phase from the speckle that appears as the high frequency and apparently random pixel-by-pixel intensity variation.

After displacement, the intensity distribution becomes

$$
I_{2}(x, y)=a(x, y)+b(x, y) \cos \left(\phi_{s}(x, y)+\Delta \varphi(x, y)\right) \text {, }
$$

where $\Delta \phi$ is the phase change in the light resulting from the displacement. The speckle fringe patterns are obtained by subtraction of a reference speckled image from image of displaced surface. In the subtraction mode, the squared 


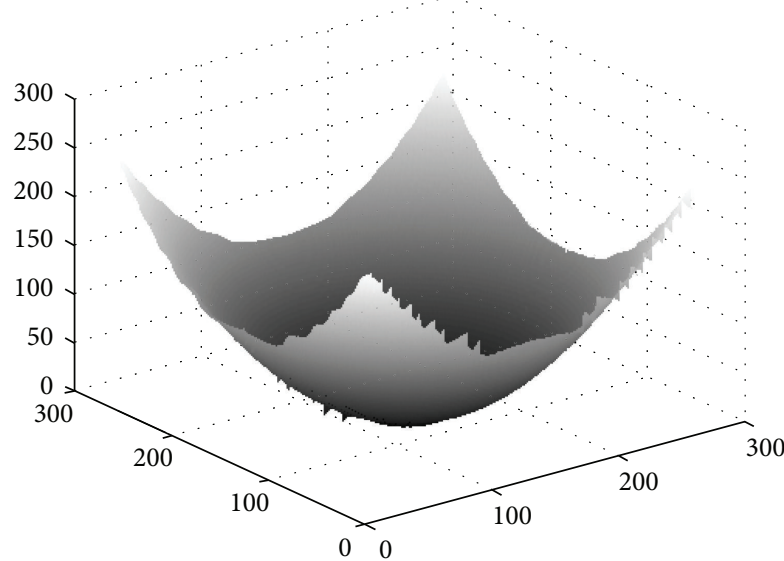

(a)

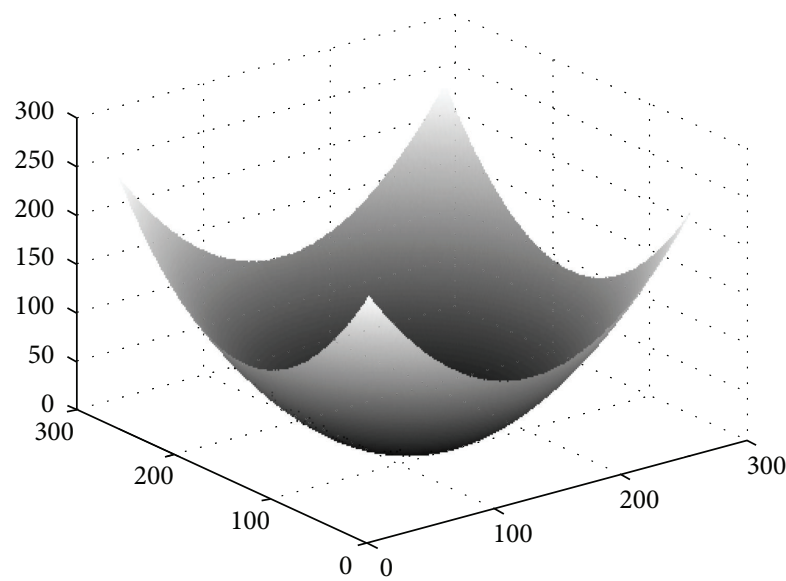

(b)

FIGURE 3: Comparison between the unwrapped phases: (a) retrieved phase; (b) test phase.

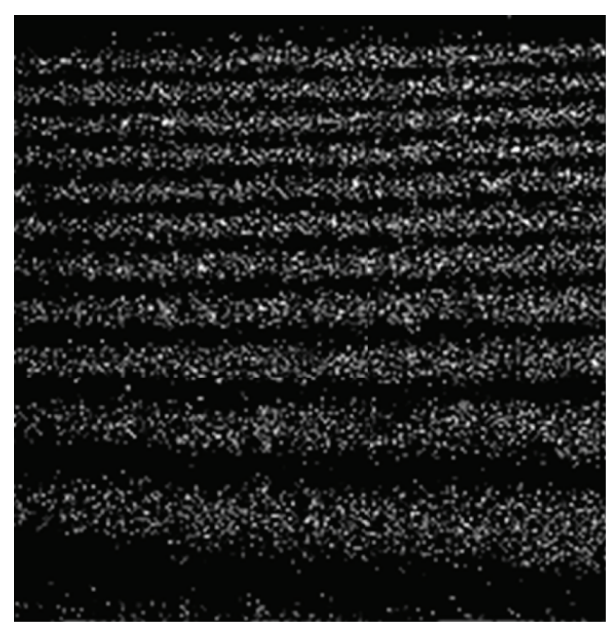

FIGURE 4: Correlation fringes pattern relative to the central section of the plate. The temperature of the plate was $325 \pm 0.2 \mathrm{~K}$. Room temperature was $295 \pm 0.2 \mathrm{~K}$.

difference between the fringe pattern before and after deformation leads to a secondary fringe pattern which is very sensitive to microstructural changes of the studied surface; if the deformation is too large, the fringe becomes too dense and may vanish due to speckle decorrelation. Speckle decorrelation limits the range of deformation that can be recorded by speckle interferometry. The effects of decorrelation are a distortion and a corresponding reduction of fringe visibility in the observation plane. We assume that the displacements are sufficiently small that speckle decorrelation effects can be ignored.

The intensity distribution in the speckle correlogram is given by

$$
I(x, y)=I_{2}(x, y)-I_{1}(x, y),
$$

$$
\begin{aligned}
I(x, y)= & 2 b(x, y) \sin \left(\frac{\Delta \varphi(x, y)}{2}\right) \\
& \times \sin \left(\phi_{s}(x, y)+\frac{\Delta \varphi(x, y)}{2}\right) .
\end{aligned}
$$

Fringes obtained are characterized by a speckle noise background; a noise reduction method must be used before the phase distribution is evaluated. The desired information containing the $\sin (\Delta \phi / 2)$ fringe term (shape of envelope modulating the random speckle term) may be rectified and filtered by appropriate computer image processing in order to remove the high frequency $\sin \left(\varphi_{s}+\Delta \phi / 2\right)$ noise.

Squaring (17) leads to

$$
\begin{aligned}
I^{2}(x, y)= & 4 b^{2}(x, y) \cdot \sin ^{2}\left(\frac{\Delta \varphi(x, y)}{2}\right) \\
& \cdot \sin ^{2}\left(\phi_{s}(x, y)+\frac{\Delta \varphi(x, y)}{2}\right) .
\end{aligned}
$$

Since $\varphi_{s}(x, y)$ changes rapidly across the speckle pattern, the ensemble average of the second sine squared term in (18) across the whole measurement area leads to

$$
\left\langle\sin ^{2}\left(\phi_{s}(x, y)+\frac{\Delta \varphi(x, y)}{2}\right)\right\rangle=\frac{1}{2} .
$$

Substituting (19) into (18) yields

$$
\begin{aligned}
I^{2}(x, y) & =2 b^{2}(x, y) \cdot \sin ^{2}\left(\frac{\Delta \varphi(x, y)}{2}\right) \\
& =b^{2}(x, y) \cdot(1-\cos (\Delta \varphi(x, y))) .
\end{aligned}
$$

Equation (20) is equivalent to the classical interferometry equation. It describes perfect correlated fringes without speckles. In practice, due to speckle decorrelation, fringe visibility is always $<1$. Fringes visualization on a monitor is sufficient for qualitative investigation. For quantitative evaluation, the temperature information has to be numerically extracted. 


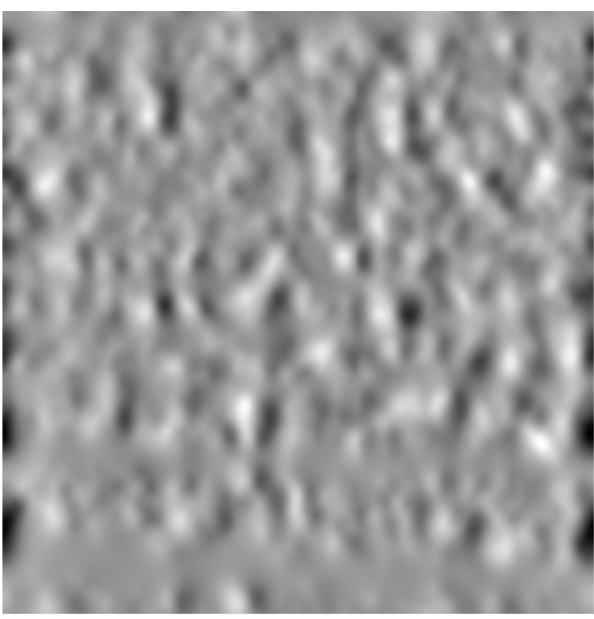

(a)

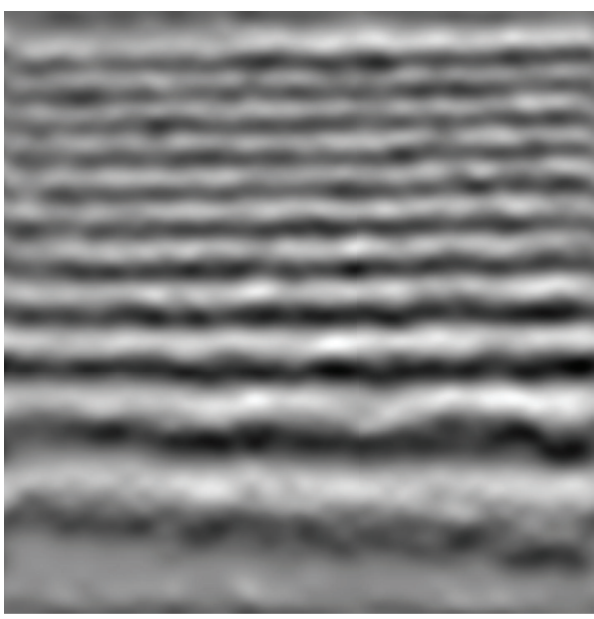

(b)

FiguRE 5: (a) Riesz component of the fringe pattern on $x$-axis; (b) Riesz component of the fringe pattern on $y$-axis.

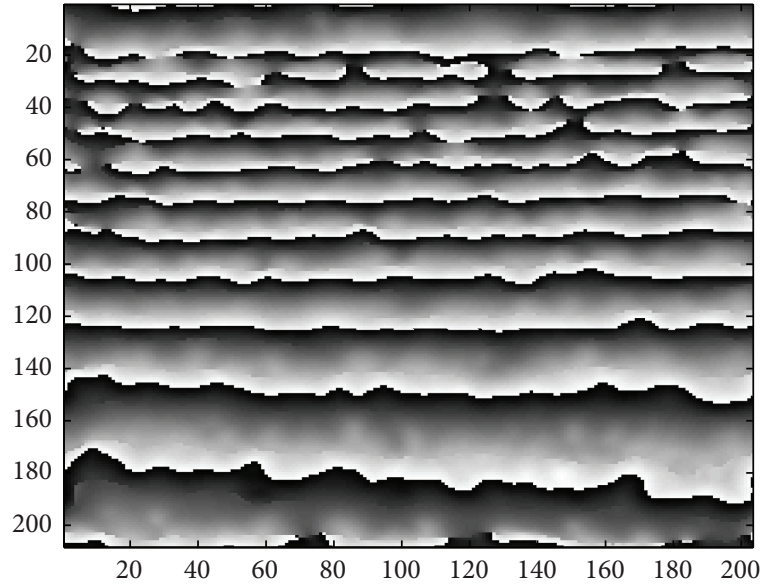

(a)

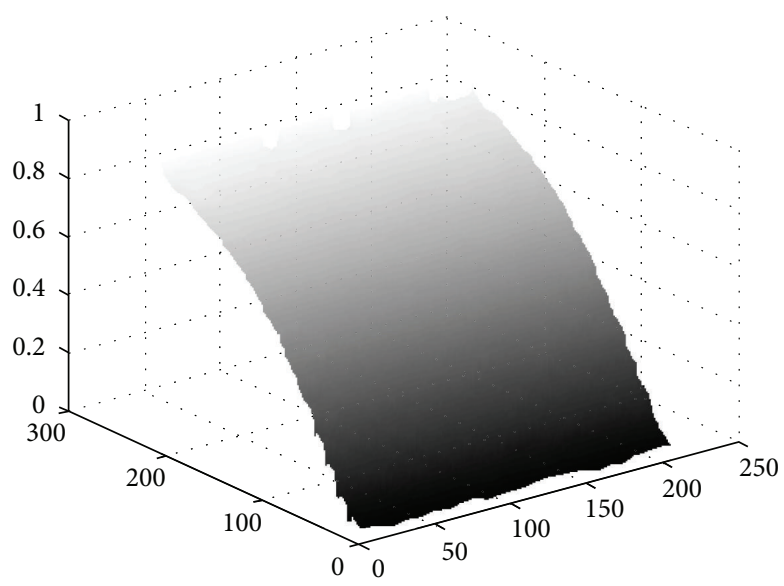

(b)

FIGURE 6: The retrieved phase by monogenic signal: (a) wrapped phase and (b) unwrapped phase.

3.2. Temperature Measurement. Now, let us consider a light beam through a test section of length $l$. The unwrapped phase is related to the variation of the refractive index of air on top of the plate surface by

$$
\Delta \varphi=\left(\frac{2 \pi \cdot l}{\lambda}\right) \cdot \Delta n
$$

where $\lambda$ is the wavelength. The change in the refractive index of air is usually related to a temperature variation through a factor approximately constant; if the temperature changes are small, the so-called Gladstone-Dale constant. The refractive index of air at $632.8 \mathrm{~nm}$ can be evaluated with greater precision by [19]

$$
n-1=\frac{0.292015 \cdot 10^{-3}}{\left(1+0.368184 \cdot 10^{-2} T\right)},
$$

where $T$ is in Celsius. This equation is based on the Gladstone-Dale is relation, with wavelength dependence calculated according to Meggers and Peters and small corrections due to Tilton. Hence

$$
\Delta n=\frac{-1.075152 .10^{-6} \cdot \Delta T}{\left(1+0.368184 \cdot 10^{-2} T\right)^{2}}
$$

From (21) and (23) we easily obtain

$$
\Delta T=\left(\frac{-1.075152 \cdot 10^{-6}}{\left(1+0.368184 \cdot 10^{-2} T\right)^{2}}\right)^{-1} \cdot\left(\frac{\lambda \cdot \Delta \varphi}{2 \pi \cdot l}\right)
$$

Owing to the linear relationship between $\Delta T$ and $\Delta \phi$, measurements of $T$ may be made automatically from the fringe pattern phase distribution using the monogenic signal phase vector. 


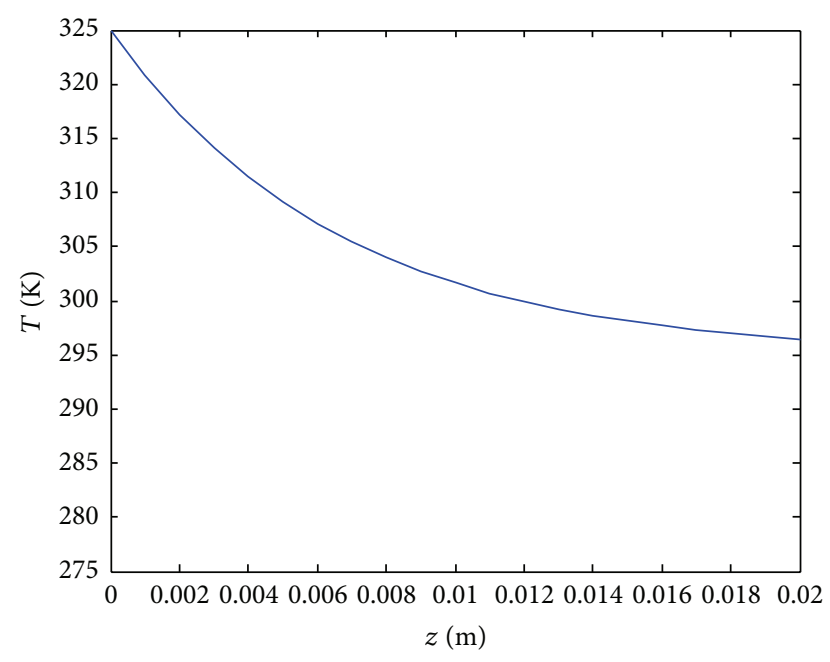

FiguRE 7: Temperature variation with distance from the plate.

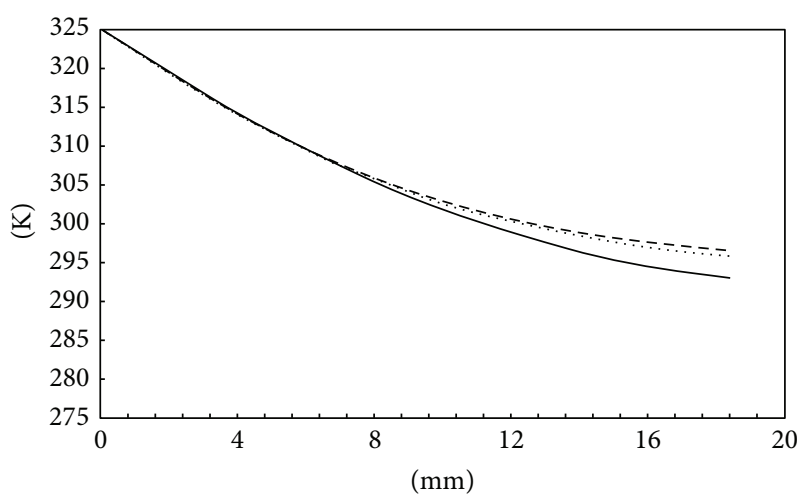

FIgURE 8: Temperature variation with distance from the plate obtained by Spagnolo and Al.

\section{Results}

4.1. Numerical Simulation. The numerical simulation consists in generating digitally a fringe pattern to verify the ability of the method to determine the phase distribution. The test phase function we used is

$$
\phi(x, y)=0.0005 \cdot\left((x-256)^{2}+(y-256)^{2}\right) .
$$

The intensity distribution of fringe pattern shown in Figure 1(a) is

$$
I(x, y)=1+0.5 \cos \phi(x, y) .
$$

We illustrated the Riesz component of the fringe pattern on $x$-axis and on $y$-axis in Figures $1(\mathrm{~b})$ and $1(\mathrm{c})$, respectively.

The wrapped retrieved phase from Riesz Transform method is plotted in Figure 2. The phase-unwrapping process which provides a continuous phase distribution over its definition domain implies suitable mathematical techniques [20], which are then needed. The unwrapped procedure consists of finding the correct field number for each phase measurement. In this work we used a fast phase unwrapping algorithm [21] that is largely immune to the presence of noise and residues and does not require strong user input. The difference between the unwrapped test phase and the unwrapped retrieved phase is depicted in Figure 3. The obtained RFSIM coefficient is 0.8250 .

4.2. Temperature Evaluation Method. To validate this phase evaluation method, real speckle correlation fringe pattern is used. The fringes pattern above a central section of a horizontal aluminum plate $(0.7 \mathrm{~cm} \times 25 \mathrm{~cm} \times 25 \mathrm{~cm})$ is shown in Figure 4 . The correlation fringe is digitized in $512 \times 512 \times$ 8 bit data, with a magnification factor such that 1 pixel $=$ $40.10^{-6} \mathrm{~m}$.

The Riesz components on $x$-axis and on $y$-axis of the correlation fringe relative to a central section of a horizontal aluminum plate are presented in Figure 5. The wrapped and unwrapped retrieved phases are depicted in Figures 6(a) and 6(b). The obtained results are in very close agreement with those obtained by the phase-shifting method. The obtained RFSIM coefficient is 0.5302 .

The temperature variation with distance from the plate as calculated from the corresponding unwrapped phase is illustrated in Figures 7 and 8 which show the temperature variation with distance from the plate obtained by [19].

\section{Conclusion}

In this work, we present a Riesz transform method for the temperature evaluation of a metal plate. The technique is based on optical phase evaluation method from which one can get the unknown temperature. The Riesz transform method provides phase distribution from a single fringe pattern with a good accuracy. This method is adequate for real time acquisition and easy to implement. We made a comparison with the results of Spagnolo and Al; we obtained results that agree relatively well with the margin of experimental error which was about $0.4 \mathrm{~K}$ near the plate surface and $0.57 \mathrm{~K}$ at a distance of about $3 \mathrm{~cm}$ from the plate. We note that our results render fairly experimental reality knowing that our method is relatively simple and inexpensive.

\section{References}

[1] V. Vit, T. Ledl, P. Psoto, and D. Roman, "Holography interferometry for measurement of temperature field in fluid," World Academy of Science and Engineering and Technology, vol. 59, pp. 2425-2428, 2011.

[2] J. Zhu, S. Huany, W. Lv, and Z. Huaichun, "Study on the measurement of using laser holographic interferometry," Combustion and Flame, vol. 49, pp. 207-219, 2011.

[3] C. Shakher and A. K. Nirala, "Measurement of temperature using speckle shearing interferometry," Applied Optics, vol. 33, no. 11, pp. 2125-2127, 1994.

[4] E. E. Keren, I. Bar-Ziv, I. Glatt, and O. Kafri, "Measurement of the temperature of the flame by moiré deflectrometry," Applied Optics, vol. 20, no. 24, pp. 4263-4266, 1981.

[5] S. Rachafi, S. Darfi, S. C. Hassani et al., "Heated plate temperature measurement by optical mirage effect," Physical and Chemical News, vol. 57, pp. 13-21, 2011. 
[6] O. Dupont, J. L. Dewandel, and J. C. Legros, "Use of electronic speckle pattern interferometry for temperature distribution measurements through liquids," Optics Letters, vol. 20, no. 17, pp. 1824-1826, 1995.

[7] K. Creath, "Phase-shifting speckle interferometry," Applied Optics, vol. 24, no. 18, pp. 3053-3058, 1985.

[8] S. Nakadate and H. Saito, "Fringe scanning speckle-pattern interferometry," Applied Optics, vol. 24, no. 18, pp. 2172-2180, 1985.

[9] A. Federico and G. H. Kaufmann, "Denoising in digital speckle pattern interferometry using wave atoms," Optics Letters, vol. 32, no. 10, pp. 1232-1234, 2007.

[10] D. L. Donoho, "De-noising by soft-thresholding," IEEE Transactions on Information Theory, vol. 41, no. 3, pp. 613-627, 1994.

[11] A. Federico and G. H. Kaufmann, "Comparative study of wavelet thresholding methods for denoising electronic speckle pattern interferometry fringes," Optical Engineering, vol. 40, no. 11, pp. 2598-2604, 2001.

[12] E. M. Barj, M. Afifi, A. A. Idrissi, K. Nassim, and S. Rachafi, "Speckle correlation fringes denoising using stationary wavelet transform. Application in the wavelet phase evaluation technique," Optics and Laser Technology, vol. 38, no. 7, pp. 506-511, 2006.

[13] M. Felsberg and G. Sommer, "The monogenic signal," IEEE Transactions on Signal Processing, vol. 49, no. 12, pp. 3136-3144, 2001.

[14] M. Riesz, "Sur les fonctions conjuguées," Mathematische Zeitschrift, vol. 27, no. 1, pp. 218-244, 1928.

[15] M. Felsberg and G. Sommer, "The monogenic scale-space: a unifying approach to phase-based image processing in scalespace," Journal of Mathematical Imaging and Vision, vol. 21, no. 1, pp. 5-26, 2004.

[16] M. Unser, D. Sage, and D. Van De Ville, "Multiresolution monogenic signal analysis using the Riesz-Laplace wavelet transform," IEEE Transactions on Image Processing, vol. 18, no. 11, pp. 2402-2418, 2009.

[17] S. Held, M. Storath, P. Massopust, and B. Forster, "Steerable wavelet frames based on the Riesz transform," IEEE Transactions on Image Processing, vol. 19, no. 3, pp. 653-667, 2010.

[18] L. Zhang, L. Zhang, and X. Mou, "RFSIM: a feature based image quality assessment metric using Riesz transforms," in Proceedings of the 17th IEEE International Conference on Image Processing (ICIP '10), pp. 321-324, Hong Kong, 2010.

[19] G. S. Spagnolo, D. Ambrosini, A. Ponticiello, and D. Paoletti, "Temperature measurement in laminar free convection using electro-optic holography," Journal de Physique III, vol. 7, no. 9, pp. 1893-1898, 1997.

[20] D. C. Ghiglia and M. D. Pritt, Two-Dimensional Phase Unwrapping: Theory, Algorithms and Software, John Wiley \& Sons, New York, NY, USA, 1998.

[21] M. A. Schofield and Y. Zhu, "Fast phase unwrapping algorithm for interferometric applications," Optics Letters, vol. 28, no. 14, pp. 1194-1196, 2003. 

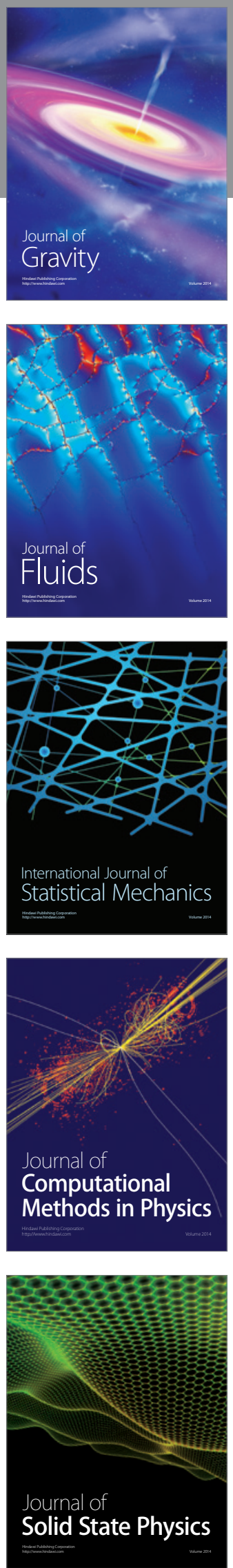

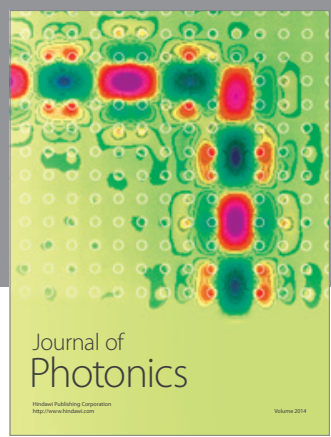

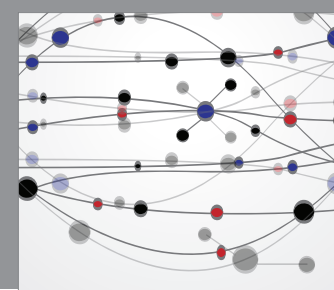

The Scientific World Journal

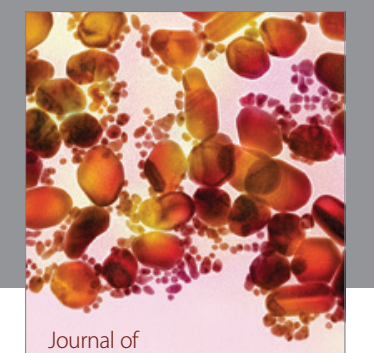

Soft Matter
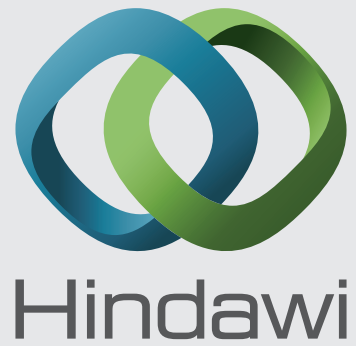

Submit your manuscripts at

http://www.hindawi.com
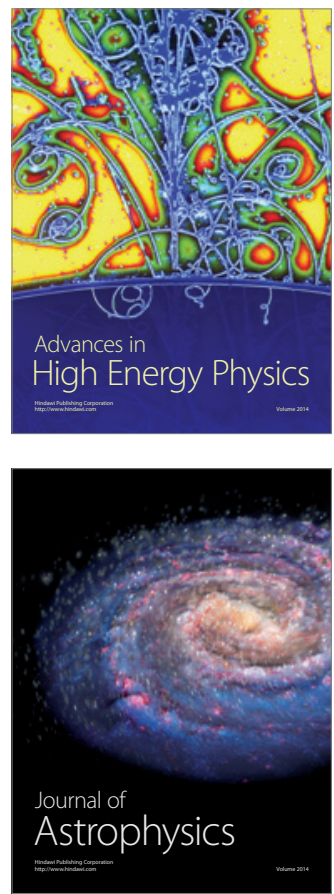
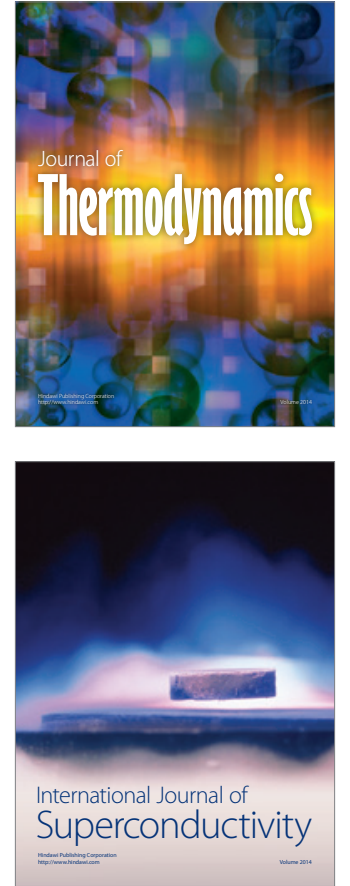
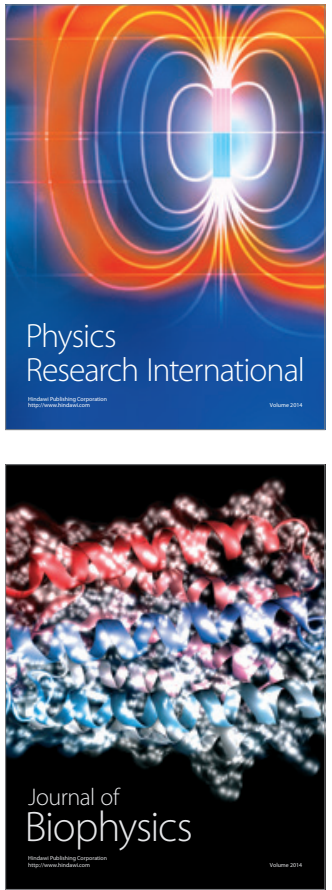
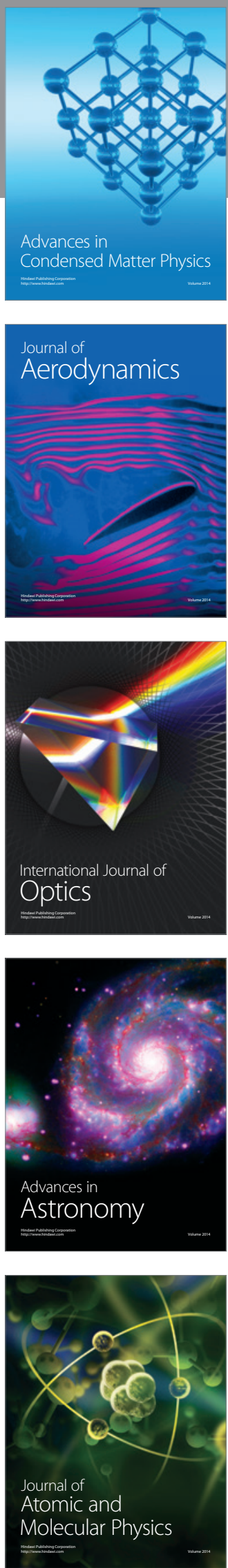